\title{
Many-Body Dispersion Correction Effects on Bulk and Surface Properties of Rutile and Anatase $\mathrm{TiO}_{2}$
}

\author{
Volker L. Deringer*, ${ }^{*},+$ and Gábor Csányi ${ }^{\dagger}$ \\ ${ }^{\dagger}$ Engineering Laboratory, University of Cambridge, Trumpington Street, Cambridge CB2 1PZ, United Kingdom \\ ${ }^{\ddagger}$ Department of Chemistry, University of Cambridge, Lensfield Road, Cambridge CB2 1EW, United Kingdom
}

\section{Supporting Information}

ABSTRACT: Titanium dioxide (titania, $\mathrm{TiO}_{2}$ ) is a widely studied material with diverse applications. Here, we explore how pairwise and many-body descriptions of van der Waals dispersion interactions perform in atomistic modeling of the two most important $\mathrm{TiO}_{2}$ polymorphs, rutile and anatase. In particular, we obtain an excellent description of both bulk structures from density-functional theory (DFT) computations with the manybody dispersion (MBD) method of Tkatchenko and co-workers coupled to an iterative Hirshfeld partitioning scheme ("HirshfeldI"). Beyond the bulk, we investigate the most important crystal surfaces, namely, rutile (110), (101), and (100) and anatase (101), (100), and (001). Dispersion has a highly anisotropic effect on the different $(h k l)$ surfaces; this directly changes the predicted nanocrystal morphology as determined from Wulff constructions. The periodic DFT+MBD method combined with Hirshfeld-I partitioning appears to be promising for future large-scale atomistic studies of this technologically important material.

\section{INTRODUCTION}

Titanium dioxide $\left(\mathrm{TiO}_{2}\right)$ is among the most widely studied metal oxides, owing both to fundamental interest and to diverse applications that range from solar cells and photocatalysis to data-storage devices. ${ }^{1-4}$ Atomistic modeling based on densityfunctional theory (DFT) has played a key part in this ${ }^{5-10}$ and often with direct links to applications: as but one example, a recent DFT study explored how the material's rich polymorphism may enable tailored band alignments for optimized photocatalysts. ${ }^{11}$ In addition, due to the abundance of highquality experimental data available, $\mathrm{TiO}_{2}$ has served as a benchmark material for many methodological studies. For example, Labat et al. compared the performance of DFT exchange-correlation functionals for the two main $\mathrm{TiO}_{2}$ polymorphs, rutile and anatase, ${ }^{7}$ and Arroyo-de Dompablo et al. explored the effect of Hubbard-type " $+U$ " corrections. Likewise, current state-of-the-art electronic-structure methods such as implementations of the random phase approximation (RPA) are commonly tested for how well they can describe the $\mathrm{TiO}_{2}$ polymorphs. ${ }^{12,13}$

Despite this progress, fundamental challenges remain when it comes to theoretical descriptions of $\mathrm{TiO}_{2}$, owing in part to its mixed ionic and covalent nature. As a prominent example, all the above DFT methods fail to reproduce the experimentally established stability ordering of the main crystalline forms of $\mathrm{TiO}_{2}$ : rutile is the stable polymorph at ambient pressure, but DFT predicts anatase to be energetically more favorable. ${ }^{6}$ In many cases, improved computational predictions can be made by augmenting traditional DFT schemes with corrections for

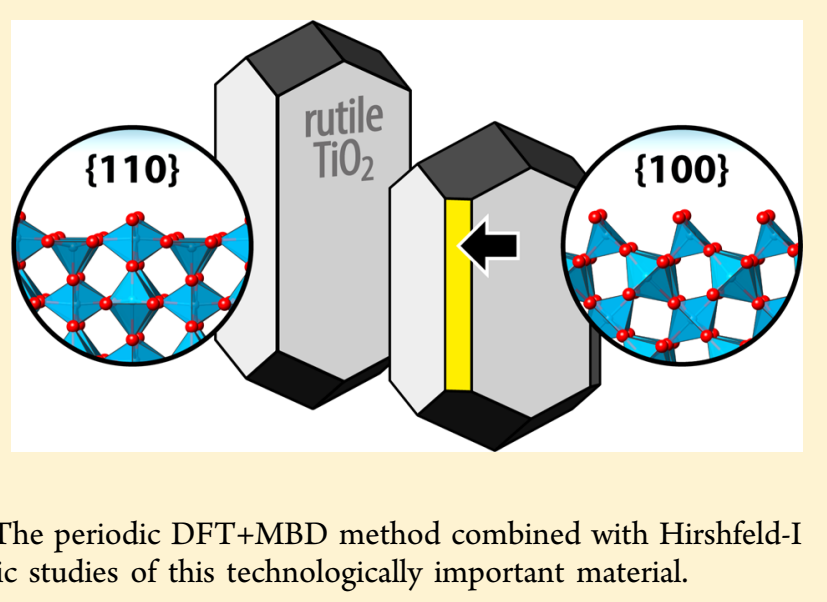

long-range van der Waals dispersion interactions ${ }^{14-17}$ or by constructing functionals which explicitly take dispersion into account. ${ }^{18} \mathrm{~A}$ popular approach is to add pairwise dispersion interaction terms after the DFT computation has been brought to self-consistency; this is computationally inexpensive and routinely done nowadays. Such pairwise corrections were shown to remedy the DFT stability ranking in several polymorphic oxides, ${ }^{19-21}$ and a similarly improved result (rutile now coming out globally more stable) has indeed been reported for $\mathrm{TiO}_{2}$ as well. ${ }^{22}$ However, this issue is not yet fully resolved, ${ }^{23}$ and we will return to it below. In time, further studies with pairwise dispersion corrections have been performed for rutile and anatase $\mathrm{e}^{23-26}$ and for possible nanostructures of $\mathrm{TiO}_{2}{ }^{27}$

Beyond pairwise terms, it has very recently become possible to compute many-body van der Waals interactions from first principles, $^{28-30}$ thereby capturing more fully the intricacies of dispersion effects in molecules and solids. It was seen, for example, that the nature and strength of these interactions in graphene multilayers or nanoribbons changes strongly with system size, and this effect cannot be described in a pairwise framework alone. ${ }^{31}$ This method has been implemented in popular plane-wave DFT codes ${ }^{32,33}$ and applied, e.g., to molecules bonding on metal surfaces ${ }^{34}$ or the polymorphism of molecular crystals. ${ }^{35-37}$

Received: July 16, 2016

Revised: $\quad$ August 26, 2016

Published: August 29, 2016 
In this work, we explore the use of pairwise and many-body dispersion schemes for rutile and anatase $\mathrm{TiO}_{2}$. To our knowledge, this represents the first such study of an oxide material using the many-body dispersion approach. Beyond the bulk structures, we investigate surfaces of both polymorphs, aiming to lay groundwork for future large-scale modeling studies of surface reactivity and nanoparticles.

\section{THEORY AND METHODS}

Dispersion Interactions: From Pairwise to Many-Body. Dispersion interactions play a key role in solids but are not accounted for in traditional DFT schemes such as the generalized gradient approximation (GGA). Here, we use two types of a posteriori dispersion corrections to DFT. The first type, of which many specific approaches exist, is built on the general idea to construct the dispersion energy contribution $E_{\text {disp }}$ by summing over all pairs of atoms $A$ and $B$ according to

$$
E_{\text {disp }}=-\frac{1}{2} \sum_{A B} f_{\text {damp }}\left(r_{A B}\right) \frac{C_{6, A B}}{\left(r_{A B}\right)^{6}}
$$

with a possible addition of higher order $\left(r^{-8}\right)$ and/or threebody terms depending on the method. ${ }^{38}$ A properly chosen damping function $f_{\text {damp }}$ ensures that the interaction term diminishes in the short-range region of covalent bonding. In the Tkatchenko-Scheffler approach ("TS" in the following), ${ }^{16}$ the $\mathrm{C}_{6}$ coefficients are obtained by scaling free-atom reference data, based on atomic volumes obtained "on the fly" from the Hirshfeld charge partitioning scheme. ${ }^{39}$ This leads to systemand environment-specific $C_{6}$ coefficients at the time of computation, despite starting from tabulated values. The TS approach has previously been used to study dye-sensitized $\mathrm{TiO}_{2}$ clusters and yielded highly reliable structural parameters, which has been an important prerequisite for subsequent higher level computations of electronic properties. ${ }^{40,41}$

Second, we go beyond pairwise terms and employ a manybody dispersion (MBD) scheme. The approach has recently been reviewed by its original authors, ${ }^{30}$ and we summarize here only the very basics. We employ the revised, range-separated MBD method of Tkatchenko and co-workers in which the selfconsistent screening (SCS) of polarizabilities is decoupled from the computation of the long-range correlation energy. This approach has hence been termed "MBD@rsSCS"; 28,29 for brevity, we simply denote it as "MBD" in the following.

The MBD method treats each atom as a fluctuating dipole, all of which are coupled to one another. Their static polarizabilities $\alpha_{A}^{\mathrm{TS}}$ and characteristic frequencies $\omega_{A}^{\mathrm{TS}}$ are again obtained by scaling tabulated values using Hirshfeld volumes, in a similar way to how the pairwise TS method determines its $C_{6}$ coefficients. These scaled quantities are then translated into the dipoles' frequency-dependent polarizability $\alpha_{A}(\omega)$

$$
\alpha_{A}(\omega)=\frac{\alpha_{A}^{\mathrm{TS}}}{1+\left(\omega / \omega_{A}^{\mathrm{TS}}\right)^{2}}
$$

Next, a set of self-consistent equations is solved (SCS step)

$$
\alpha_{A}^{\mathrm{SCS}}(i \omega)=\alpha_{A}(i \omega)+\alpha_{A}(i \omega) \sum_{B \neq A} \mathbf{T}_{\mathrm{SR}, A B} \alpha_{B}^{\mathrm{SCS}}(i \omega)
$$

where $\mathbf{T}_{\mathrm{SR}}$ is the short-range part of the dipole-dipole coupling tensor which is range separated into short-range and long-range contributions by means of a damping function. This function, in turn, is controlled by the atoms' van der Waals radii, and these are again scaled from tabulated values using Hirshfeld output. In the definition of this damping function, a single free parameter $\beta$ is introduced and multiplied onto the sum of the van der Waals radii; optimized values have been determined for different cases (see below). ${ }^{29,42}$ This gives the long-range contribution to the dipole-dipole coupling, denoted $\mathbf{T}_{\mathrm{LR}}$.

Together with the values for $\alpha_{A}^{\mathrm{SCS}}(\omega)$ (eq 3), which are collected in the matrix $\mathbf{A}(\omega)$, the expression for the MBD energy is then

$$
E_{\mathrm{MBD}}=\frac{1}{2 \pi} \int_{0}^{\infty} d \omega \operatorname{Tr}\left[\ln \left(\mathbf{1}-\mathbf{A}(\omega) \mathbf{T}_{\mathrm{LR}}\right)\right]
$$

which is computed "on the fly" and added to the Kohn-Sham energy. The extension to periodic systems (that is, a reciprocalspace implementation) has very recently been accomplished by Bučko and co-workers. ${ }^{33}$ Furthermore, expressions for the gradients have been derived and implemented to obtain MBDcorrected forces on atoms, which makes geometry optimization possible. $^{33}$

Charge Partitioning. Both TS and MBD are based on a Hirshfeld partitioning scheme for the DFT charge density: this assigns a volume to each atom in the system, and the ratio of this to the free-atom volume is used to scale the dispersion coefficients (TS) as well as polarizabilities, characteristic frequencies, and van der Waals radii (MBD).

While the traditional Hirshfeld method $^{39}$ experiences difficulties in describing ionic solids, a more recently proposed iterative Hirshfeld method (dubbed Hirshfeld-I) ${ }^{43}$ has been found well suitable for this task. ${ }^{44-46}$ Rather than using isolated, neutral atoms as reference, the Hirshfeld-I approach employs the initially determined atomic charges as reference state for the next partitioning step, and this cycle is repeated until the charges converge below a given threshold. ${ }^{43-46}$ Indeed, for the oxide anion in the optimized rutile structure, our computations yield a charge of $-0.36 e$ using traditional Hirshfeld partitioning but a much more reasonable $-1.06 e$ based on Hirshfeld-I. The latter result is well in line with Mulliken charges obtained previously for the oxide ion in rutile; these ranged between $-1.02 e$ and $-1.18 e$ when using different local basis sets and exchange-correlation functionals (from the simple LDA to the hybrid PBE0). ${ }^{7}$ We remark that this step is, conceptually, the analogue of finding improved dispersion coefficients for use with the Grimme scheme when dealing with ionic rather than covalent systems. ${ }^{22,23}$

Computational Details. DFT computations were performed using the projector augmented-wave (PAW) method ${ }^{47}$ as implemented in the Vienna ab initio Simulation Package (VASP 5.4.1). ${ }^{48-51}$ Titanium $3 \mathrm{~s} 3 \mathrm{p} 4 \mathrm{~s} 3 \mathrm{~d}$ and oxygen $2 \mathrm{~s} 2 \mathrm{p}$ levels were expanded into plane waves with a cutoff of $500 \mathrm{eV}$. The convergence criterion for electronic SCF iterations was $\Delta E$ $<10^{-6} \mathrm{eV}$. Crystal structures were initially optimized at various volume increments such as to minimize forces on atoms below $10^{-3} \mathrm{eV} \AA^{-1}$, followed by a fit to the Birch-Murnaghan equation of state ${ }^{52}$ and a second optimization at the soobtained equilibrium volume. Reciprocal space was sampled on dense Monkhorst-Pack grids, ${ }^{53}$ sized $12 \times 12 \times 18$ for bulk rutile, $16 \times 16 \times 4$ for bulk anatase, and adjusted accordingly for surface models. In a test computation for bulk rutile with a finer $24 \times 24 \times 36$ grid, the Kohn-Sham energy changed by less than $0.002 \mathrm{meV}$ per formula unit and the MBD energy by less than $0.2 \mathrm{meV}$ per formula unit. 
Effects of exchange and correlation were modeled in the GGA after Perdew, Burke, and Ernzerhof (PBE), ${ }^{54}$ on which the current $\mathrm{MBD}$ implementation is based. ${ }^{29,33}$ It was previously seen that for ionic solids, higher rung hybrid DFT schemes show good performance particularly when coupled with (pairwise) dispersion corrections; see, for example, studies of $\mathrm{NaCl}$ (HSE06+TS) ${ }^{55}$ or rutile and anatase $\mathrm{TiO}_{2}$ $(\mathrm{PBE} 0+\mathrm{D} 2){ }^{23}$ However, with future large-scale simulations of surface and nanoparticle models in mind, these hybrid functionals are not computationally feasible at this time, and we focus on their economic GGA counterparts instead. To apply dispersion corrections as discussed above, we use the implementations of Bučko and co-workers for the $\mathrm{TS}^{56}$ $\mathrm{MBD}^{33}$ and Hirshfeld-I methods, ${ }^{45,46}$ all as included in the release version of VASP. For the combined use of MBD with Hirshfeld-I partitioning, an optimized scaling parameter of $\beta=$ 0.82 has thereby been found and implemented by Bučko. ${ }^{42}$

\section{RESULTS AND DISCUSSION}

Bulk Structures. The first test for the quality of a computational method is how well it can describe the equilibrium crystal structures. For both rutile and anatase, results from powder neutron diffraction at very low temperature $(15 \mathrm{~K})$ are available ${ }^{57}$ and constitute the "gold standard" experimental reference. Our computational results are compared against these benchmarks in Table 1.

Table 1. Lattice Parameters and Atomic Positions of Rutile and Anatase $\mathrm{TiO}_{2}$

\begin{tabular}{llll}
\multicolumn{1}{c}{ rutile $^{a}$} & \multicolumn{1}{c}{$a(\AA)$} & \multicolumn{1}{c}{$c(\AA)$} & \multicolumn{1}{c}{$x$} \\
expt. $^{57}(15 \mathrm{~K})$ & $4.58666(4)$ & $2.95407(3)$ & $0.30469(6)$ \\
$\mathrm{PBE}$ & $4.651(+1.4 \%)$ & $2.969(+0.5 \%)$ & 0.3051 \\
$\mathrm{PBE}+\mathrm{TS}$ & $4.613(+0.6 \%)$ & $2.970(+0.5 \%)$ & 0.3044 \\
PBE+MBD & $4.609(+0.5 \%)$ & $2.953(-0.0 \%)$ & 0.3037 \\
$\quad$ anatase $^{b}$ & $a(\AA)$ & $c(\AA)$ & $z$ \\
expt. $^{57}(15 \mathrm{~K})$ & $3.78216(3)$ & $9.50226(12)$ & $0.16675(4)$ \\
PBE & $3.804(+0.6 \%)$ & $9.720(+2.3 \%)$ & 0.1687 \\
PBE+TS & $3.788(+0.2 \%)$ & $9.666(+1.7 \%)$ & 0.1680 \\
PBE+MBD & $3.783(+0.0 \%)$ & $9.590(+0.9 \%)$ & 0.1668
\end{tabular}

${ }^{a}$ Space group $P 4_{2} / m n m$; titanium on $2 a(0,0,0)$; oxygen on $4 f(x, x$, $0) .{ }^{b}$ Space group $I 4_{1} / a m d$; titanium on $4 b(0,1 / 4,3 / 8)$; oxygen on $8 e$ $(0,1 / 4, z)$.

The uncorrected PBE functional overestimates the lattice parameters, as expected; our data agree with a previous study at the same level of theory. ${ }^{23}$ The pairwise TS scheme lowers the error and describes the rutile structure highly satisfactorily; for anatase, it yields an excellent $a$ parameter $(\Delta a=+0.2 \%)$ but overshoots $c$ by almost $2 \%$. A significant step further is made when moving on to PBE+MBD computations. Only with the latter method we are able to reproduce all lattice parameters to within less than $1 \%$; for the least problematic cases, rutile $c$ and anatase $a, \mathrm{PBE}+\mathrm{MBD}$ gives results identical to experiment within any reasonable accuracy limit. We reiterate that the experimental data in ref 57 have been gathered at very low temperature, where thermal lattice expansion is negligible, and thus, these experiments do allow for side-by-side comparison with the computed minimum structures.

Overall, the largest deviations from experiment are seen for the anatase $c$ parameter (Figure 1a), which is not surprising: anatase is less densely packed than rutile and thus more challenging to describe by DFT. This particular structural
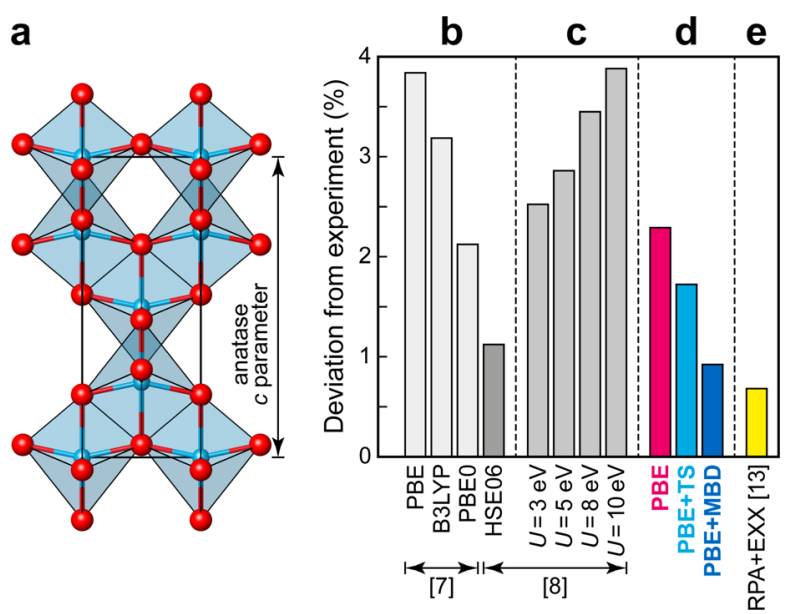

Figure 1. (a) Bulk structure of anatase $\mathrm{TiO}_{2}$, optimized at the $\mathrm{PBE}$ $+\mathrm{MBD}$ level of theory; the unit cell is indicated. All structural drawings in this work were created with VESTA. ${ }^{58}(\mathrm{~b}-\mathrm{e})$ Deviation of predicted anatase $c$ parameters from the experimental value in ref 57, grouped according to different exemplarily chosen computational methods. Data in $b$ and $c$ taken from refs 7 and 8 ; the rightmost value (yellow) is from a very recent RPA study. ${ }^{13}$ See text for discussion.

parameter thus appears to be a suitable and sensitive test, as noted before, ${ }^{25}$ and we use it to now place our results in a larger context. In Figure $1 \mathrm{~b}-\mathrm{e}$, we assess the performance of different computational methods that are commonly applied to $\mathrm{TiO}_{2}$. The first group of data (Figure $1 \mathrm{~b}$ ) surveys the role of post-GGA functionals, displaying results from a study based on the linear combination of atomic orbitals (LCAO), ${ }^{7}$ comparing GGA (PBE) and hybrid (B3LYP, PBE0) functionals (light gray bars). The PBE value quoted there deviates more strongly from experiment $(+3.8 \%)$ than the present result $(+2.3 \%$; Table 1$)$; the difference is likely due to different computational parameters and basis sets but does not affect the direct comparison with the hybrid functionals using the same settings. Moving from PBE to the hybrid PBE0 almost cut the error in half; $^{7}$ clearly, an admixture of Hartree-Fock exchange can improve the result. The last entry in Figure $1 \mathrm{~b}$ (dark gray) confirms this: the latter has been obtained with the HSE06 functional in a plane-wave-based DFT study ${ }^{8}$ (that is, with a computational framework more similar to what is used here), and indeed, it describes the anatase $c$ parameter very well. However, the use of hybrid functionals such as HSE06 in this framework is much more demanding than PBE+MBD.

The data reproduced in Figure 1c assesses the effect of Hubbard-type corrections $(\mathrm{PBE}+U$; from ref 8). These corrections are frequently applied for defect computations and off-stoichiometric $\mathrm{Ti}-\mathrm{O}$ phases. Regarding structural data, however, adding $U$ terms progressively worsens the result (a similar effect had been observed for the rutile structure in ref 8). Again, we will hereafter focus on the pure PBE functional as the underlying electronic-structure method.

Our own computations (Figure 1d) emphasize the progressive improvement of the PBE result (magenta) when adding TS (light blue) or MBD (dark blue) corrections. The latter, as already noted in Table 1, brings the error below $1 \%$ even for this most challenging lattice parameter. For completeness, we include the result of a current state-of-theart method, namely, the RPA with exact exchange (EXX) in Figure 1e. ${ }^{13}$ This one performs slightly better than MBD for the anatase $c$ parameter but again is much more demanding. 
Indeed, as discussed in ref 13 , the computations had to be performed nonself-consistently, whereas self-consistent RPAEXX studies are currently restricted to even much smaller systems (see, e.g., a study of noble-gas dimers in ref 59).

It is also important to compare the present results to those from other popular methods for treating dispersion. To do so, we focus on the computed unit-cell volumes which provide an integral indicator for the relevance of dispersion (thought of as additional "spring forces" between atoms). A systematic study has been reported by Grimme and co-workers for the widely used "D2"/"D3" family of dispersion correction techniques, including computations with optimized dispersion coefficients adapted to the ionic nature of the material (dubbed "D3*”). ${ }^{23}$ This comparison is performed in Figure 2.

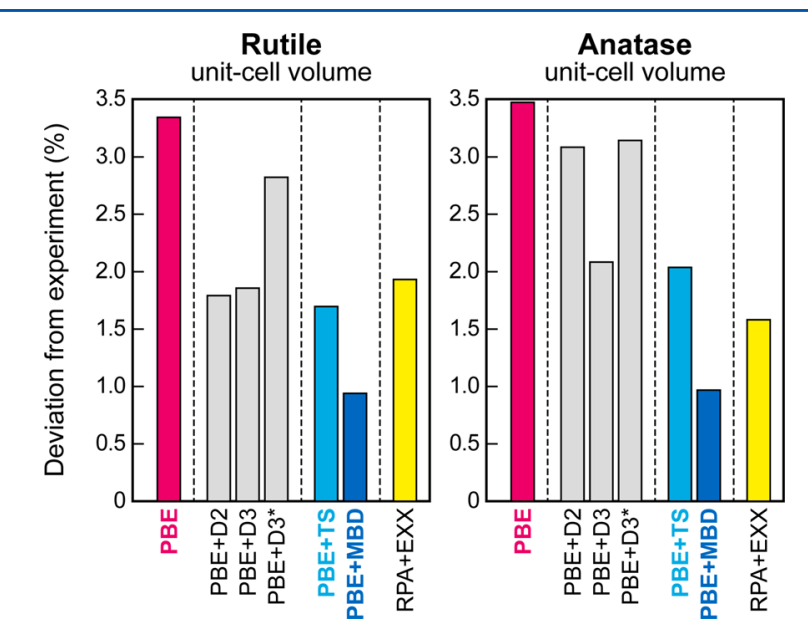

Figure 2. Deviation of predicted unit-cell volumes from experiment (ref 57), comparing different methods for dispersion correction. D2, D3, and D3* data (gray) are taken from Grimme and co-workers; ${ }^{23}$ the rightmost values (yellow) are from a very recent RPA study. ${ }^{13}$

As noted in ref 23 already, it is surprising that the (physically better motivated) D3* correction does not improve over its counterpart with standard parameters. Our results from the TS scheme (which is conceptually best comparable to D3*, because it also uses optimized $C_{6}$ coefficients) are in line with the previous $\mathrm{PBE}+\mathrm{D} 3$ data. The MBD approach improves the situation further, leading to a deviation from experiment below $1 \%$ for both polymorphs. It is also quite interesting that the many-body correction outperforms RPA+EXX for both unit-cell volumes (Figure 2).

Note that Grimme and co-workers reported more detailed data than discussed here, including comparison of dispersion corrections to the revPBE functional and for the hybrid PBE0 and revPBE0 schemes. ${ }^{23}$ However, revPBE did not visibly improve upon $\mathrm{PBE},{ }^{23}$ and the hybrid functionals are computationally too demanding at present to be feasible for large-scale surface models, as said above. We hence limit our comparison to the PBE functional which is the one on which the MBD method is built.

We finally investigate the aforementioned energy ranking and find that neither method employed here accomplishes this. The uncorrected PBE method with the present computational settings makes anatase more stable than rutile by $9.1 \mathrm{~kJ} \mathrm{~mol}^{-1}$; dispersion corrections lower this difference to 3.4 (+TS) and $3.9(+\mathrm{MBD}) \mathrm{kJ} \mathrm{mol}^{-1}$, respectively, but the inverted trend remains. Indeed, it has been argued that the previously observed stability ranking at the $\mathrm{PBE}+\mathrm{D} 2$ level (ref 22) may have been fortuitous. ${ }^{23}$ Subsequent studies found a combination of $+U$ and $+\mathrm{D} 2$ corrections to be successful in reproducing the polymorph ordering, ${ }^{26}$ but doubts regarding fortuitous error cancellations may remain, especially given the very small difference between the crystalline forms. A recently described way out would be RPA+EXX, which yields $2.6 \mathrm{~kJ} \mathrm{~mol}^{-1}$ in favor of rutile, ${ }^{13}$ in excellent agreement with an experimental value of $2.6 \pm 0.4 \mathrm{~kJ} \mathrm{~mol}^{-1}$ (ref 60$)$. We conclude that even state-of-theart dispersion corrections are unable to reproduce the rutile-vsanatase stability ordering as long as they are based on GGAlevel computations. This does not at all impede their usefulness for studying either of the polymorphs, as we will show below for the surface energies.

Compression Behavior. Figure 3 shows the unit-cell volumes as a function of increasing external pressure, again
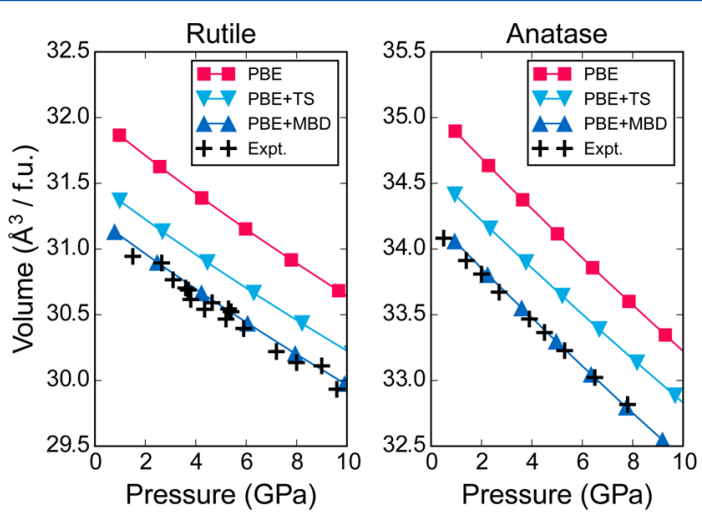

Figure 3. Equations of state (course of unit-cell volume with pressure) for rutile and anatase $\mathrm{TiO}_{2}$. Experimental data for rutile and anatase are taken from refs 61 and 62, respectively. Lines connecting data points are guides to the eye.

computed at three different levels of theory. Comparison with high-pressure experiments reveals that $\mathrm{PBE}+\mathrm{MBD}$ not only captures the equilibrium structures (Table 1) but the course of cell volumes over the entire range where experimental data are available. The slope of the data is steeper for anatase than for rutile, reflecting its easier compressibility. At even higher pressures, transformations to different polymorphs will occur; these are not the topic of this work but should constitute an interesting direction for future studies.

We furthermore evaluated the bulk moduli of both compounds (Table 2). The PBE results are consistently too low, and it was previously seen that $+U$-type corrections predict the material to be even softer. ${ }^{8}$ Both the TS and the MBD corrections, by contrast, yield bulk moduli within the experimental error for both polymorphs. The overall dispersion contribution to bulk moduli is visible but small; in this regard, $\mathrm{TiO}_{2}$ clearly differs from less ionic oxides such as $\mathrm{TeO}_{2}$ (where

Table 2. Bulk Moduli of Rutile and Anatase $\mathrm{TiO}_{2}$

$\begin{array}{lcl} & \text { rutile } B(\mathrm{GPa}) & \text { anatase } B(\mathrm{GPa}) \\ \text { expt. (ref 61) } & 211(7) & \\ \text { expt. (ref 63) } & 230(20) & \\ \text { expt. (ref 62) } & & 178(1) \\ \text { expt. (ref 64) } & & 179(2) \\ \text { PBE } & 200 & 169 \\ \text { PBE+TS } & 212 & 176 \\ \text { PBE+MBD } & 210 & 168\end{array}$


the addition of dispersion corrections to PBE roughly doubles the computed bulk modulus and thereby brings it much closer to the experimental benchmark). ${ }^{21}$

Surface Structure of Rutile (110). The $\mathrm{TiO}_{2}$ surfaces have been studied previously in seminal theoretical contributions, ${ }^{65-69}$ and thus, we focus here on the influence of dispersion interactions. The best studied among the titania surfaces is rutile (110) (Figure $4 \mathrm{a}$ and $4 \mathrm{~b}$ ). For this case, highquality experimental structure data are available; it has thus served as a benchmark before to assess dispersion corrections. ${ }^{23}$

Indeed, the experiments on rutile (110) themselves have sparked some controversy, and only recently has a consensus been reached by comparing two complementary surfaceanalytical techniques: ${ }^{70}$ on one hand, previous quantitative low-energy electron diffraction (LEED) data ${ }^{71}$ were reanalyzed
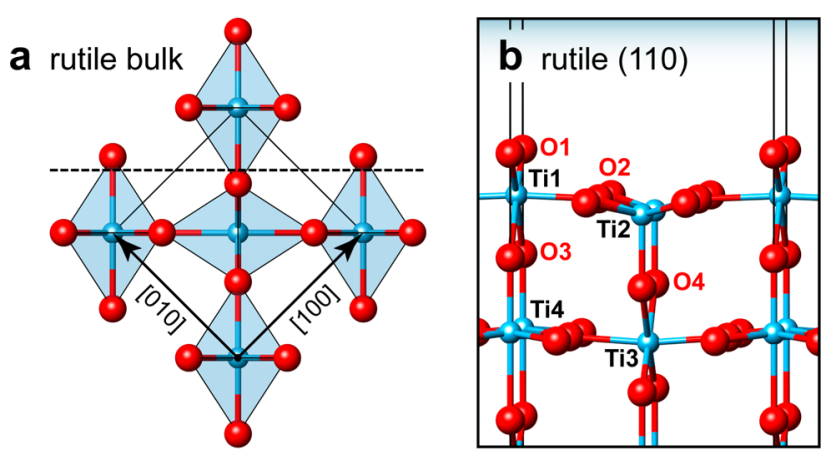

C surface relaxation

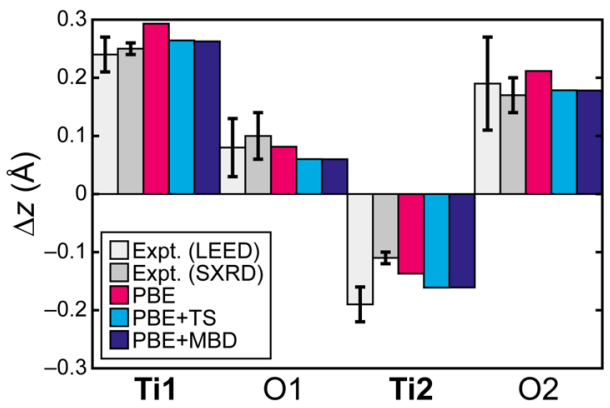

d subsurface relaxation

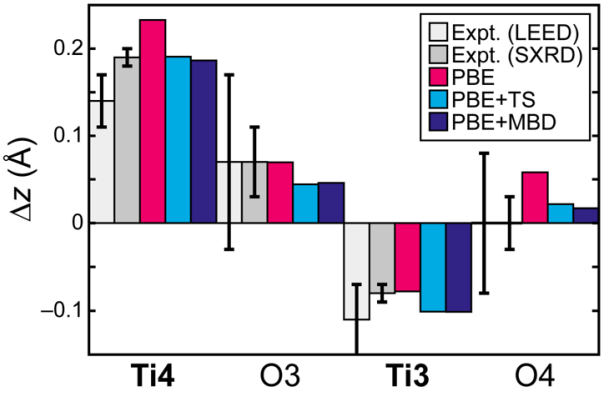

Figure 4. (a) Unit cell of rutile $\mathrm{TiO}_{2}$ with the lowest energy $\{110\}$ cleavage plane indicated. (b) Side view of the rutile (110) surface with atomic labeling as in previous literature. ${ }^{23,70}$ In the unrelaxed surface model, $\mathrm{Ti} 2$ and $\mathrm{O} 2$ lie in the same horizontal plane, whereas in the relaxed structure (as shown here at the $\mathrm{PBE}+\mathrm{MBD}$ level), the undercoordinated Ti2 atom has moved inward $(\Delta z<0)$ and $\mathrm{O} 2$ has relaxed outward $(\Delta z>0)$, respectively. (c) Quantitative atomic relaxations at the surface from experiments (ref 70 ) and theory (this work). (d) Same for the subsurface atoms. Ordering of the graphs corresponds to the nomenclature in panel $b$. to achieve higher precision; on the other hand, surface X-ray diffraction (SXRD) had been applied a few years earlier. ${ }^{72}$ Results from both methods are in good agreement (Figure $4 \mathrm{c}$ and $4 \mathrm{~d}$ ), with the exception of the undercoordinated Ti2 atom and the subsurface $\mathrm{Ti} 4$ for which the experimental relaxations differ by more than their respective standard deviations. ${ }^{70}$ It is also apparent that the experimental error bars are very large for some atoms, especially for the subsurface oxygen species.

Our computational results, overall, are in sound agreement with the experimental benchmarks. For Til, the atom with the strongest outward relaxations, the reference error bars are small, and this case offers itself as a particularly suitable test. Here, PBE (magenta in Figure $4 c$ and $4 d$ ) overestimates the outward motion, while both the TS and the MBD results coincide perfectly with the experimental values. Another interesting case is the oxygen atom $\mathrm{O} 4$, directly below the undercoordinated titanium atom; both experiments predict a zero relaxation, which is much better mirrored by the TS and MBD results than by the outcome of pure PBE. Notably, both types of dispersion corrections (light versus dark blue in Figure $4 \mathrm{c}$ and $4 \mathrm{~d}$ ) arrive at almost the same quantitative relaxations, and this holds for the $\mathrm{Ti}$ as well as for the $\mathrm{O}$ atoms at the surface.

Surface Energies. We now move on to a comparative study of the most important crystal surfaces for both polymorphs (Figure 5) and focus on their stabilities as predicted by

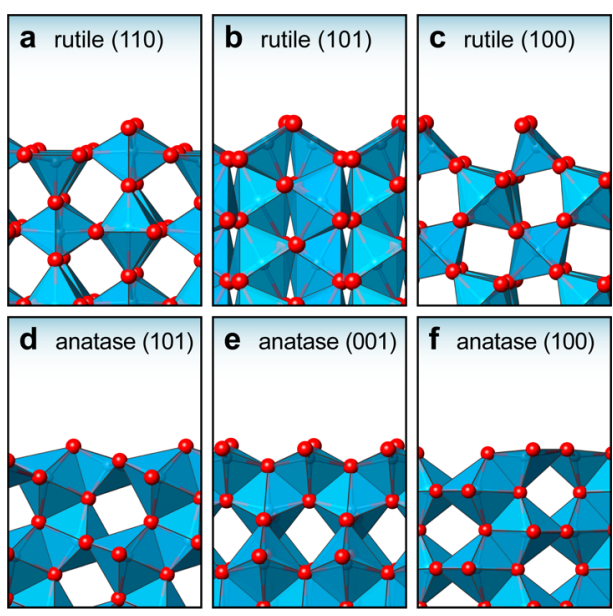

Figure 5. $(a-c)$ Structures of the most important rutile $\mathrm{TiO}_{2}$ surfaces, optimized at the PBE+MBD level of theory. Coordination polyhedra have been drawn to emphasize the presence of undercoordinated (5fold) environments at the surfaces. (d-f) Same but for anatase.

computations. The relevant quantity, namely, the surface energy $\gamma$, is obtained as

$$
\gamma=\frac{1}{2 A}\left[E^{\text {slab }}-N \times E^{\text {bulk }}\right]
$$

where $E$ denotes the DFT-computed total energies for the surface slab (per cell) and for the optimized bulk structure (per formula unit), respectively. The slab model contains $N$ formula units of $\mathrm{TiO}_{2}$ and exposes an area of $A$ at the top and bottom (hence the factor two in the denominator). We use slab thicknesses that had previously been found to lead to sufficient convergence (ref 68), vacuum regions of $20 \AA$ to separate periodic replicae, and a force tolerance of $10^{-2} \mathrm{eV} \AA^{-1}$ for optimizing surface models. All optimized structural models are provided as Supporting Information. 
The effect of dispersion corrections to DFT on computed surface energies has been probed earlier for oxides and higher chalcogenides. ${ }^{73-75}$ Here, in line with this previous work, it is observed that all surfaces increase their energies significantly as a consequence of dispersion. Importantly, however, the data in Table 3 suggest that the influence of dispersion on the various

Table 3. Computed Surface Energies of Rutile and Anatase $\mathrm{TiO}_{2}$

\begin{tabular}{|c|c|c|c|c|c|c|}
\hline \multirow[b]{2}{*}{ rutile } & \multicolumn{3}{|c|}{ unrelaxed $\left(\mathrm{J} \mathrm{m}^{-2}\right)$} & \multicolumn{3}{|c|}{ relaxed $\left(\mathrm{J} \mathrm{m}^{-2}\right)$} \\
\hline & $(110)$ & $(100)$ & $\overline{(101)}$ & (110) & (100) & $(101)$ \\
\hline PBE & 1.34 & 1.42 & 1.64 & 0.42 & 0.60 & 0.95 \\
\hline $\mathrm{PBE}+\mathrm{TS}$ & 1.65 & 1.73 & 1.91 & 0.77 & 0.95 & 1.27 \\
\hline \multirow[t]{2}{*}{$\mathrm{PBE}+\mathrm{MBD}$} & 1.68 & 1.77 & 1.93 & 0.80 & 1.00 & 1.31 \\
\hline & \multicolumn{3}{|c|}{ unrelaxed $\left(\mathrm{J} \mathrm{m}^{-2}\right)$} & \multicolumn{3}{|c|}{ relaxed $\left(\mathrm{J} \mathrm{m}^{-2}\right)$} \\
\hline anatase & (101) & (100) & (001) & (101) & (100) & $(001)$ \\
\hline PBE & 1.17 & 1.46 & 1.08 & 0.46 & 0.57 & 1.02 \\
\hline $\mathrm{PBE}+\mathrm{TS}$ & 1.39 & 1.69 & 1.30 & 0.71 & 0.82 & 1.25 \\
\hline $\mathrm{PBE}+\mathrm{MBD}$ & 1.43 & 1.74 & 1.31 & 0.75 & 0.86 & 1.26 \\
\hline
\end{tabular}

$\mathrm{TiO}_{2}$ crystal surfaces is not uniform but rather specific to the structure. We evaluate this more systematically by calculating the percent change in surface energy compared to the pure PBE result (Figure 6). Rutile (110), for example, comes out almost double as expensive to form when MBD corrections are switched on; this effect is much less pronounced for rutile (101).
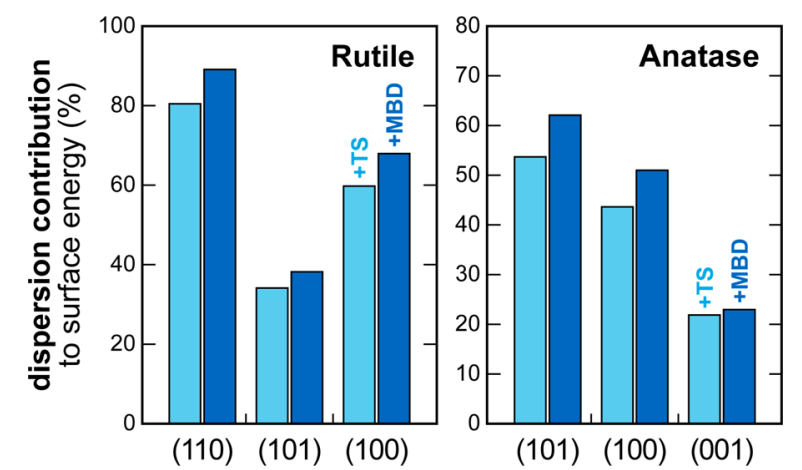

Figure 6. Changes in computed relaxed surface energies due to dispersion, relative to the uncorrected PBE result (derived from the absolute values; cf. Table 3).

The incorrect ordering of the bulk polymorphs has been mentioned above, and it is finally interesting to assess whether this error influences the stability ranking of the surfaces. The $\mathrm{PBE}+\mathrm{MBD}$ method predicts a difference of $\Delta E$ (rutile-anatase) $=+0.04 \mathrm{meV} / \mathrm{fu}$, whereas the experimental value is -0.03 $\mathrm{meV} / \mathrm{fu} ;{ }^{60}$ thus, we assume an additional $0.07 \mathrm{meV} / \mathrm{fu}$ error for the bulk energies to enter the surface energy calculation. Inserting this into eq 5 and normalizing by the number of atoms and surface area, we estimate a deviation of roughly $\Delta \gamma$ $\approx 0.001 \mathrm{~J} \mathrm{~m}^{-2}$. This is very small compared to the absolute values, which are on the order of $\gamma \approx 1 \mathrm{~J} \mathrm{~m}^{-2}$ (Table 3).

Wulff Constructions. We performed Wulff constructions $^{76,77}$ to determine the equilibrium crystal shapes as predicted at the various levels of theory (Figure 7). For both polymorphs, adding dispersion has a qualitative effect on the Wulff shapes, as observed before for lead chalcogenides. ${ }^{75}$ In the present case of $\mathrm{TiO}_{2}$, this is most pronounced for rutile, a Rutile
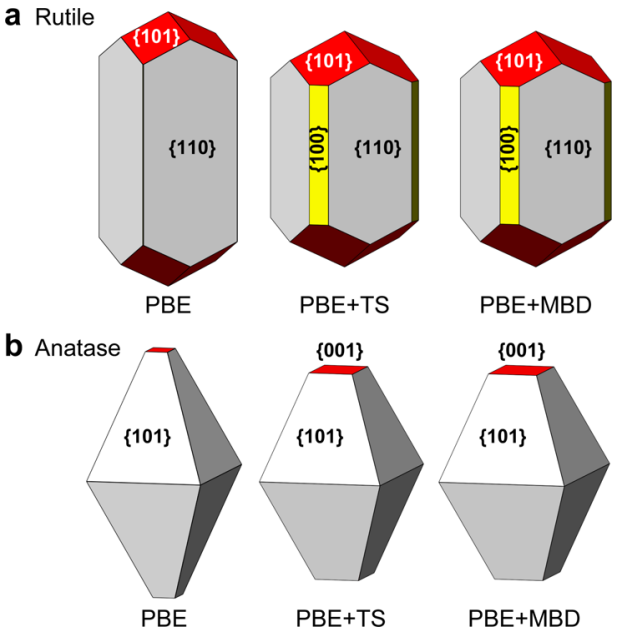

Figure 7. (a) Equilibrium crystal morphologies of rutile $\mathrm{TiO}_{2}$ as predicted using the Wulff construction. Note the presence of $\{100\}$ facets as soon as dispersion corrections are switched on. The pairwise and many-body results, however, are barely distinguishable to the naked eye. Note that the absolute sizes of the polyhedra are arbitrary; it is the relative ratio of surface areas that is of interest here. (b) Same for anatase $\mathrm{TiO}_{2}$.

where the $\{100\}$ faces (yellow) are either visible in the polyhedra or not. Following the Gibbs-Wulff theorem, the $\{100\}$ faces can compete with their $\{110\}$ counterparts only if $\gamma_{110} / \gamma_{100}>1 / \sqrt{ } 2$. The PBE surface energies are just on the verge of this criterion being fulfilled (in fact, a minuscule $\{100\}$ contribution is made to the Wulff polyhedron but this is invisible to the naked eye). When using dispersion corrections, $\gamma_{110}$ for rutile increases more strongly than $\gamma_{100}$ (Figure 6), and the above criterion is indeed satisfied.

Again, there is little difference between the results obtained using pairwise and many-body corrections-this is in line with the underlying surface-energy data (Table 3). Hence, it appears that for surface studies and investigations of pristine morphologies the TS correction should be well suited. The situation may well change when exploring molecular adsorbates (which can strongly influence morphology; refs 66 and 78) and reactive species on the different surfaces. Indeed, it has previously been seen that MBD contributions are influential for an accurate computational description of adsorbates on metal surfaces while at the same time being efficient enough to facilitate large-scale simulations. ${ }^{34}$ The present, optimized $\mathrm{TiO}_{2}$ surface models at the $\mathrm{PBE}+\mathrm{MBD}$ level may well provide a starting point for future endeavors of this type.

\section{CONCLUSIONS}

van der Waals dispersion has a notable effect on the bulk structures and surface stabilities of rutile and anatase $\mathrm{TiO}_{2}$. Including appropriate dispersion corrections hence much improves the outcome of DFT-GGA simulations in these regards while at the same time providing a very attractive ratio of accuracy and computational cost. The bulk lattice parameters obtained from the MBD method are in excellent agreement with experiment and rival the outcome of much more expensive RPA simulations. $\mathrm{TiO}_{2}$ crystal surfaces, likewise, experience strong dispersion effects, but for the clean surfaces studied here the pairwise TS corrections already capture the largest share of the interactions. The dispersion contribution is very different for the various $(h k l)$ surfaces and polymorphs, and thus, the 
computed equilibrium crystal shapes are visibly affected. Our results have provided general insight into the nature of van der Waals interactions in ionic materials, and they suggest PBE $+\mathrm{MBD}$ as an economic and powerful method to treat rutile and anatase $\mathrm{TiO}_{2}$ in future work.

\section{ASSOCIATED CONTENT}

\section{S Supporting Information}

The Supporting Information is available free of charge on the ACS Publications website at DOI: 10.1021/acs.jpcc.6b07141.

Details regarding input files and computed energies (PDF)

Structural data in $X Y Z$ format for optimized bulk and surface models at the PBE, PBE+TS, and PBE+MBD levels of theory (ZIP)

\section{AUTHOR INFORMATION}

\section{Corresponding Author}

*E-mail: vld24@cam.ac.uk. Phone: +44 12237 48582. Fax: +44 1223332662.

\section{Notes}

The authors declare no competing financial interest.

\section{ACKNOWLEDGMENTS}

V.L.D. gratefully acknowledges a postdoctoral fellowship from the Alexander von Humboldt Foundation. This work used the ARCHER UK National Supercomputing Service (http://www. archer.ac.uk), access to which was granted via support for the UKCP consortium (EPSRC Grant EP/K014560/1). Data Access Statement: Structural and energetic data for this publication are provided as Supporting Information. Furthermore, original output files have been deposited at http://dx.doi. org/10.17863/CAM.1731.

\section{REFERENCES}

(1) Fujishima, A.; Honda, K. Electrochemical Photolysis of Water at a Semiconductor Electrode. Nature 1972, 238, 37-38.

(2) O’Regan, B.; Grätzel, M. A Low-Cost, High-Efficiency Solar Cell Based on Dye-Sensitized Colloidal $\mathrm{TiO}_{2}$ Films. Nature 1991, 353, 737-740.

(3) Fujishima, A.; Rao, T. N.; Tryk, D. A. Titanium Dioxide Photocatalysis. J. Photochem. Photobiol., C 2000, 1, 1-21.

(4) Szot, K.; Rogala, M.; Speier, W.; Klusek, Z.; Besmehn, A.; Waser, R. $\mathrm{TiO}_{2}-$ a Prototypical Memristive Material. Nanotechnology 2011, 22, 254001.

(5) Glassford, K. M.; Chelikowsky, J. R. Structural and Electronic Properties of Titanium Dioxide. Phys. Rev. B: Condens. Matter Mater. Phys. 1992, 46, 1284-1298.

(6) Muscat, J.; Swamy, V.; Harrison, N. M. First-Principles Calculations of the Phase Stability of $\mathrm{TiO}_{2}$. Phys. Rev. B: Condens. Matter Mater. Phys. 2002, 65, 224112.

(7) Labat, F.; Baranek, P.; Domain, C.; Minot, C.; Adamo, C. Density Functional Theory Analysis of the Structural and Electronic Properties of $\mathrm{TiO}_{2}$ Rutile and Anatase Polytypes: Performances of Different Exchange-Correlation Functionals. J. Chem. Phys. 2007, 126, 154703.

(8) Arroyo-de Dompablo, M. E.; Morales-García, A.; Taravillo, M. $\mathrm{DFT}+\mathrm{U}$ Calculations of Crystal Lattice, Electronic Structure, and Phase Stability under Pressure of $\mathrm{TiO}_{2}$ Polymorphs. J. Chem. Phys. 2011, 135, 054503.

(9) Mei, Z.-G.; Wang, Y.; Shang, S.-L.; Liu, Z.-K. First-Principles Study of Lattice Dynamics and Thermodynamics of $\mathrm{TiO}_{2}$ Polymorphs. Inorg. Chem. 2011, 50, 6996-7003.
(10) Curnan, M. T.; Kitchin, J. R. Investigating the Energetic Ordering of Stable and Metastable $\mathrm{TiO}_{2}$ Polymorphs Using DFT+U and Hybrid Functionals. J. Phys. Chem. C 2015, 119, 21060-21071.

(11) Buckeridge, J.; Butler, K. T.; Catlow, C. R. A.; Logsdail, A. J.; Scanlon, D. O.; Shevlin, S. A.; Woodley, S. M.; Sokol, A. A.; Walsh, A. Polymorph Engineering of $\mathrm{TiO}_{2}$ : Demonstrating How Absolute Reference Potentials Are Determined by Local Coordination. Chem. Mater. 2015, 27, 3844-3851.

(12) Spreafico, C.; VandeVondele, J. The Nature of Excess Electrons in Anatase and Rutile from Hybrid DFT and RPA. Phys. Chem. Chem. Phys. 2014, 16, 26144-26152.

(13) Patrick, C. E.; Thygesen, K. S. Hubbard- U Corrected Hamiltonians for Non-Self-Consistent Random-Phase Approximation Total-Energy Calculations: A Study of $\mathrm{ZnS}, \mathrm{TiO}_{2}$, and $\mathrm{NiO}$. Phys. Rev. B: Condens. Matter Mater. Phys. 2016, 93, 035133.

(14) Wu, Q.; Yang, W. Empirical Correction to Density Functional Theory for van Der Waals Interactions. J. Chem. Phys. 2002, 116, 515.

(15) Grimme, S. Semiempirical GGA-Type Density Functional Constructed with a Long-Range Dispersion Correction. J. Comput. Chem. 2006, 27, 1787-1799.

(16) Tkatchenko, A.; Scheffler, M. Accurate Molecular Van Der Waals Interactions from Ground-State Electron Density and FreeAtom Reference Data. Phys. Rev. Lett. 2009, 102, 73005.

(17) Grimme, S. Density Functional Theory with London Dispersion Corrections. Wiley Interdiscip. Rev. Comput. Mol. Sci. 2011, 1, 211-228.

(18) Berland, K.; Cooper, V. R.; Lee, K.; Schröder, E.; Thonhauser, T.; Hyldgaard, P.; Lundqvist, B. I. Van Der Waals Forces in Density Functional Theory: A Review of the vdW-DF Method. Rep. Prog. Phys. 2015, 78, 066501.

(19) Allen, J. P.; Scanlon, D. O.; Parker, S. C.; Watson, G. W. Tin Monoxide: Structural Prediction from First Principles Calculations with van Der Waals Corrections. J. Phys. Chem. C 2011, 115, 1991619924.

(20) Guńka, P. A.; Dranka, M.; Piechota, J.; Żukowska, G. Z.; Zalewska, A.; Zachara, J. $\mathrm{As}_{2} \mathrm{O}_{3}$ Polymorphs: Theoretical Insight into Their Stability and Ammonia Templated Claudetite II Crystallization. Cryst. Growth Des. 2012, 12, 5663-5670.

(21) Deringer, V. L.; Stoffel, R. P.; Dronskowski, R. Thermochemical Ranking and Dynamic Stability of $\mathrm{TeO}_{2}$ Polymorphs from $\mathrm{Ab}$ Initio Theory. Cryst. Growth Des. 2014, 14, 871-878.

(22) Conesa, J. C. The Relevance of Dispersion Interactions for the Stability of Oxide Phases. J. Phys. Chem. C 2010, 114, 22718-22726.

(23) Moellmann, J.; Ehrlich, S.; Tonner, R.; Grimme, S. A DFT-D Study of Structural and Energetic Properties of $\mathrm{TiO}_{2}$ Modifications. J. Phys.: Condens. Matter 2012, 24, 424206.

(24) Reckien, W.; Janetzko, F.; Peintinger, M. F.; Bredow, T. Implementation of Empirical Dispersion Corrections to Density Functional Theory for Periodic Systems. J. Comput. Chem. 2012, 33, 2023-2031.

(25) Albuquerque, A. R.; Garzim, M. L.; Santos, I. M. G. d.; Longo, V.; Longo, E.; Sambrano, J. R. DFT Study with Inclusion of the Grimme Potential on Anatase $\mathrm{TiO}_{2}$ : Structure, Electronic, and Vibrational Analyses. J. Phys. Chem. A 2012, 116, 11731-11735.

(26) Zhu, T.; Gao, S.-P. The Stability, Electronic Structure, and Optical Property of $\mathrm{TiO}_{2}$ Polymorphs. J. Phys. Chem. C 2014, 118, 11385-11396.

(27) Forrer, D.; Vittadini, A. 2D vs. 3D Titanium Dioxide: Role of Dispersion Interactions. Chem. Phys. Lett. 2011, 516, 72-75.

(28) Tkatchenko, A.; DiStasio, R. A.; Car, R.; Scheffler, M. Accurate and Efficient Method for Many-Body van Der Waals Interactions. Phys. Rev. Lett. 2012, 108, 236402.

(29) Ambrosetti, A.; Reilly, A. M.; DiStasio, R. A.; Tkatchenko, A. Long-Range Correlation Energy Calculated from Coupled Atomic Response Functions. J. Chem. Phys. 2014, 140, 18A508.

(30) DiStasio, R. A.; Gobre, V. V.; Tkatchenko, A. Many-Body van Der Waals Interactions in Molecules and Condensed Matter. J. Phys.: Condens. Matter 2014, 26, 213202. 
(31) Gobre, V. V.; Tkatchenko, A. Scaling Laws for van Der Waals Interactions in Nanostructured Materials. Nat. Commun. 2013, 4, 2341.

(32) Reilly, A. M.; Tkatchenko, A. Understanding the Role of Vibrations, Exact Exchange, and Many-Body van Der Waals Interactions in the Cohesive Properties of Molecular Crystals. J. Chem. Phys. 2013, 139, 024705.

(33) Bučko, T.; Lebègue, S.; Gould, T.; Ángyán, J. G. Many-Body Dispersion Corrections for Periodic Systems: An Efficient Reciprocal Space Implementation. J. Phys.: Condens. Matter 2016, 28, 045201.

(34) Maurer, R. J.; Ruiz, V. G.; Tkatchenko, A. Many-Body Dispersion Effects in the Binding of Adsorbates on Metal Surfaces. J. Chem. Phys. 2015, 143, 102808.

(35) DiStasio, R. A.; von Lilienfeld, O. A.; Tkatchenko, A. Collective Many-Body van Der Waals Interactions in Molecular Systems. Proc. Natl. Acad. Sci. U. S. A. 2012, 109, 14791-14795.

(36) Marom, N.; DiStasio, R. A.; Atalla, V.; Levchenko, S.; Reilly, A. M.; Chelikowsky, J. R.; Leiserowitz, L.; Tkatchenko, A. Many-Body Dispersion Interactions in Molecular Crystal Polymorphism. Angew. Chem., Int. Ed. 2013, 52, 6629-6632.

(37) Reilly, A. M.; Tkatchenko, A. Role of Dispersion Interactions in the Polymorphism and Entropic Stabilization of the Aspirin Crystal. Phys. Rev. Lett. 2014, 113, 055701.

(38) Grimme, S.; Antony, J.; Ehrlich, S.; Krieg, H. A Consistent and Accurate $\mathrm{Ab}$ Initio Parametrization of Density Functional Dispersion Correction (DFT-D) for the 94 Elements H-Pu. J. Chem. Phys. 2010, 132, 154104.

(39) Hirshfeld, F. L. Bonded-Atom Fragments for Describing Molecular Charge Densities. Theor. Chim. Acta 1977, 44, 129-138.

(40) Marom, N.; Moussa, J. E.; Ren, X.; Tkatchenko, A.; Chelikowsky, J. R. Electronic Structure of Dye-Sensitized $\mathrm{TiO}_{2}$ Clusters from Many-Body Perturbation Theory. Phys. Rev. B: Condens. Matter Mater. Phys. 2011, 84, 245115.

(41) Marom, N.; Körzdörfer, T.; Ren, X.; Tkatchenko, A.; Chelikowsky, J. R. Size Effects in the Interface Level Alignment of Dye-Sensitized $\mathrm{TiO}_{2}$ Clusters. J. Phys. Chem. Lett. 2014, 5, 2395-2401.

(42) Bučko, T. Private Communication.

(43) Bultinck, P.; Van Alsenoy, C.; Ayers, P. W.; Carbó-Dorca, R. Critical Analysis and Extension of the Hirshfeld Atoms in Molecules. J. Chem. Phys. 2007, 126, 144111.

(44) Vanpoucke, D. E. P.; Bultinck, P.; Van Driessche, I. Extending Hirshfeld-I to Bulk and Periodic Materials. J. Comput. Chem. 2013, 34, 405-417.

(45) Bučko, T.; Lebègue, S.; Hafner, J.; Ángyán, J. G. Improved Density Dependent Correction for the Description of London Dispersion Forces. J. Chem. Theory Comput. 2013, 9, 4293-4299.

(46) Bučko, T.; Lebègue, S.; Ángyán, J. G.; Hafner, J. Extending the Applicability of the Tkatchenko-Scheffler Dispersion Correction via Iterative Hirshfeld Partitioning. J. Chem. Phys. 2014, 141, 034114.

(47) Blöchl, P. E. Projector Augmented-Wave Method. Phys. Rev. B: Condens. Matter Mater. Phys. 1994, 50, 17953-17979.

(48) Kresse, G.; Hafner, J. Ab Initio Molecular Dynamics for Liquid Metals. Phys. Rev. B: Condens. Matter Mater. Phys. 1993, 47, 558-561.

(49) Kresse, G.; Furthmüller, J. Efficient Iterative Schemes for Ab Initio Total-Energy Calculations Using a Plane-Wave Basis Set. Phys. Rev. B: Condens. Matter Mater. Phys. 1996, 54, 11169-11186.

(50) Kresse, G.; Furthmüller, J. Efficiency of Ab-Initio Total Energy Calculations for Metals and Semiconductors Using a Plane-Wave Basis Set. Comput. Mater. Sci. 1996, 6, 15-50.

(51) Kresse, G.; Joubert, D. From Ultrasoft Pseudopotentials to the Projector Augmented-Wave Method. Phys. Rev. B: Condens. Matter Mater. Phys. 1999, 59, 1758-1775.

(52) Birch, F. Finite Elastic Strain of Cubic Crystals. Phys. Rev. 1947, $71,809-824$.

(53) Monkhorst, H. J.; Pack, J. D. Special Points for Brillouin-Zone Integrations. Phys. Rev. B 1976, 13, 5188-5192.

(54) Perdew, J. P.; Burke, K.; Ernzerhof, M. Generalized Gradient Approximation Made Simple. Phys. Rev. Lett. 1996, 77, 3865-3868.
(55) Zhang, G.-X.; Tkatchenko, A.; Paier, J.; Appel, H.; Scheffler, M. Van Der Waals Interactions in Ionic and Semiconductor Solids. Phys. Rev. Lett. 2011, 107, 245501.

(56) Bučko, T.; Lebègue, S.; Hafner, J.; Ángyán, J. G. TkatchenkoScheffler van Der Waals Correction Method with and without SelfConsistent Screening Applied to Solids. Phys. Rev. B: Condens. Matter Mater. Phys. 2013, 87, 064110.

(57) Burdett, J. K.; Hughbanks, T.; Miller, G. J.; Richardson, J. W.; Smith, J. V. Structural-Electronic Relationships in Inorganic Solids: Powder Neutron Diffraction Studies of the Rutile and Anatase Polymorphs of Titanium Dioxide at 15 and 295 K. J. Am. Chem. Soc. 1987, 109, 3639-3646.

(58) Momma, K.; Izumi, F. VESTA 3 for Three-Dimensional Visualization of Crystal, Volumetric and Morphology Data. J. Appl. Crystallogr. 2011, 44, 1272-1276.

(59) Nguyen, N. L.; Colonna, N.; de Gironcoli, S. Ab Initio SelfConsistent Total-Energy Calculations within the EXX/RPA Formalism. Phys. Rev. B: Condens. Matter Mater. Phys. 2014, 90, 045138.

(60) Ranade, M. R.; Navrotsky, A.; Zhang, H. Z.; Banfield, J. F.; Elder, S. H.; Zaban, A.; Borse, P. H.; Kulkarni, S. K.; Doran, G. S.; Whitfield, H. J. Energetics of Nanocrystalline $\mathrm{TiO}_{2}$. Proc. Natl. Acad. Sci. U. S. A. 2002, 99 (Suppl 2), 6476-6481.

(61) Ming, L.; Manghnani, M. H. Isothermal Compression of $\mathrm{TiO}_{2}$ (Rutile) under Hydrostatic Pressure to 106 Kbar. J. Geophys. Res. 1979, 84, 4777.

(62) Swamy, V.; Dubrovinsky, L. S. Bulk Modulus of Anatase. J. Phys. Chem. Solids 2001, 62, 673-675.

(63) Gerward, L.; Olsen, J. S. Post-Rutile High-Pressure Phases in $\mathrm{TiO}_{2}$. J. Appl. Crystallogr. 1997, 30, 259-264.

(64) Arlt, T.; Bermejo, M.; Blanco, M. A.; Gerward, L.; Jiang, J. Z.; Staun Olsen, J.; Recio, J. M. High-Pressure Polymorphs of Anatase $\mathrm{TiO}_{2}$. Phys. Rev. B: Condens. Matter Mater. Phys. 2000, 61, 1441414419.

(65) Ramamoorthy, M.; Vanderbilt, D.; King-Smith, R. D. FirstPrinciples Calculations of the Energetics of Stoichiometric $\mathrm{TiO}_{2}$ Surfaces. Phys. Rev. B: Condens. Matter Mater. Phys. 1994, 49, 16721-16727.

(66) Barnard, A. S.; Zapol, P. Effects of Particle Morphology and Surface Hydrogenation on the Phase Stability of $\mathrm{TiO}_{2}$. Phys. Rev. B: Condens. Matter Mater. Phys. 2004, 70, 235403.

(67) Perron, H.; Domain, C.; Roques, J.; Drot, R.; Simoni, E.; Catalette, H. Optimisation of Accurate Rutile $\mathrm{TiO}_{2}$ (110), (100), (101) and (001) Surface Models from Periodic DFT Calculations. Theor. Chem. Acc. 2007, 117, 565-574.

(68) Labat, F.; Baranek, P.; Adamo, C. Structural and Electronic Properties of Selected Rutile and Anatase $\mathrm{TiO}_{2}$ Surfaces: $\mathrm{An} \mathrm{Ab}$ Initio Investigation. J. Chem. Theory Comput. 2008, 4, 341-352.

(69) Esch, T. R.; Gadaczek, I.; Bredow, T. Surface Structures and Thermodynamics of Low-Index of Rutile, Brookite and Anatase - A Comparative DFT Study. Appl. Surf. Sci. 2014, 288, 275-287.

(70) Busayaporn, W.; Torrelles, X.; Wander, A.; Tomić, S.; Ernst, A.; Montanari, B.; Harrison, N. M.; Bikondoa, O.; Joumard, I.; Zegenhagen, J.; et al. Geometric Structure of $\mathrm{TiO}_{2}(110)(1 \times 1)$ : Confirming Experimental Conclusions. Phys. Rev. B: Condens. Matter Mater. Phys. 2010, 81, 153404.

(71) Lindsay, R.; Wander, A.; Ernst, A.; Montanari, B.; Thornton, G.; Harrison, N. M. Revisiting the Surface Structure of $\mathrm{TiO}_{2}$ (110): A Quantitative Low-Energy Electron Diffraction Study. Phys. Rev. Lett. 2005, 94, 246102.

(72) Cabailh, G.; Torrelles, X.; Lindsay, R.; Bikondoa, O.; Joumard, I.; Zegenhagen, J.; Thornton, G. Geometric Structure of $\mathrm{TiO}_{2}$ (110) $(1 \times 1)$ : Achieving Experimental Consensus. Phys. Rev. B: Condens. Matter Mater. Phys. 2007, 75, 241403.

(73) Deringer, V. L.; Dronskowski, R. DFT Studies of Pristine Hexagonal $\mathrm{Ge}_{1} \mathrm{Sb}_{2} \mathrm{Te}_{4}(0001), \mathrm{Ge}_{2} \mathrm{Sb}_{2} \mathrm{Te}_{5}(0001)$, and $\mathrm{Ge}_{1} \mathrm{Sb}_{4} \mathrm{Te}_{7}(0001)$ Surfaces. J. Phys. Chem. C 2013, 117, 15075-15089.

(74) Ončák, M.; Włodarczyk, R.; Sauer, J. Water on the $\mathrm{MgO}(001)$ Surface: Surface Reconstruction and Ion Solvation. J. Phys. Chem. Lett. 2015, 6, 2310-2314. 
(75) Deringer, V. L.; Dronskowski, R. Stabilities and Reconstructions of Clean PbS and PbSe Surfaces: DFT Results and the Role of Dispersion Forces. J. Phys. Chem. C 2016, 120, 8813-8820.

(76) Wulff, G. Zur Frage Der Geschwindigkeit Des Wachstums Und Der Auflösung von Krystallflächen [in German]. Z. Kryst. Miner. 1901, 34, 449-530.

(77) Moll, N.; Kley, A.; Pehlke, E.; Scheffler, M. GaAs Equilibrium Crystal Shape from First Principles. Phys. Rev. B: Condens. Matter Mater. Phys. 1996, 54, 8844-8855.

(78) Barnard, A. S.; Curtiss, L. A. Prediction of $\mathrm{TiO}_{2}$ Nanoparticle Phase and Shape Transitions Controlled by Surface Chemistry. Nano Lett. 2005, 5, 1261-1266. 